\title{
Several Higher Differentiation Formulas of Special Functions
}

\author{
Junjie Zhao \\ Qingdao University of Science \\ and Technology \\ China
}

\author{
Xiquan Liang \\ Qingdao University of Science \\ and Technology \\ China
}

\author{
Li Yan \\ Qingdao University of Science \\ and Technology \\ China
}


(7) If $n \geq 1$ and $f$ is differentiable $n$ times on $Z$, then $f$ is differentiable on $Z$.

(8) $\square^{n}$ is differentiable on $\mathbb{R}$.

(9) If $x \in Z$, then (the function $\sin )^{\prime}(Z)(2)(x)=-\sin x$.

(10) If $x \in Z$, then (the function $\sin )^{\prime}(Z)(3)(x)=-\cos x$.

(11) If $x \in Z$, then (the function $\sin )^{\prime}(Z)(n)(x)=\sin \left(x+\frac{n \cdot \pi}{2}\right)$.

(12) If $x \in Z$, then (the function $\cos )^{\prime}(Z)(2)(x)=-\cos x$.

(13) If $x \in Z$, then (the function $\cos )^{\prime}(Z)(3)(x)=\sin x$.

(14) If $x \in Z$, then (the function $\cos )^{\prime}(Z)(n)(x)=\cos \left(x+\frac{n \cdot \pi}{2}\right)$.

(15) If $f_{1}$ is differentiable $n$ times on $Z$ and $f_{2}$ is differentiable $n$ times on $Z$, then $\left(f_{1}+f_{2}\right)^{\prime}(Z)(n)=f_{1}{ }^{\prime}(Z)(n)+f_{2}{ }^{\prime}(Z)(n)$.

(16) If $f_{1}$ is differentiable $n$ times on $Z$ and $f_{2}$ is differentiable $n$ times on $Z$, then $\left(f_{1}-f_{2}\right)^{\prime}(Z)(n)=f_{1}{ }^{\prime}(Z)(n)-f_{2}{ }^{\prime}(Z)(n)$.

(17) If $f_{1}$ is differentiable $n$ times on $Z$ and $f_{2}$ is differentiable $n$ times on $Z$ and $i \leq n$, then $\left(f_{1}+f_{2}\right)^{\prime}(Z)(i)=f_{1}{ }^{\prime}(Z)(i)+f_{2}{ }^{\prime}(Z)(i)$.

(18) If $f_{1}$ is differentiable $n$ times on $Z$ and $f_{2}$ is differentiable $n$ times on $Z$ and $i \leq n$, then $\left(f_{1}-f_{2}\right)^{\prime}(Z)(i)=f_{1}{ }^{\prime}(Z)(i)-f_{2}{ }^{\prime}(Z)(i)$.

(19) If $f_{1}$ is differentiable $n$ times on $Z$ and $f_{2}$ is differentiable $n$ times on $Z$, then $f_{1}+f_{2}$ is differentiable $n$ times on $Z$.

(20) If $f_{1}$ is differentiable $n$ times on $Z$ and $f_{2}$ is differentiable $n$ times on $Z$, then $f_{1}-f_{2}$ is differentiable $n$ times on $Z$.

(21) If $f$ is differentiable $n$ times on $Z$, then $(r f)^{\prime}(Z)(n)=r f^{\prime}(Z)(n)$.

(22) If $f$ is differentiable $n$ times on $Z$, then $r f$ is differentiable $n$ times on $Z$.

(23) If $f$ is differentiable on $Z$, then $f^{\prime}(Z)(1)=f_{\uparrow Z}^{\prime}$.

(24) If $n \geq 1$ and $f$ is differentiable $n$ times on $Z$, then $f^{\prime}(Z)(1)=f_{i Z}^{\prime}$.

(25) If $x \in Z$, then $(r$ (the function $\sin ))^{\prime}(Z)(n)(x)=r \cdot \sin \left(x+\frac{n \cdot \pi}{2}\right)$.

(26) If $x \in Z$, then $(r$ (the function $\cos ))^{\prime}(Z)(n)(x)=r \cdot \cos \left(x+\frac{n \cdot \pi}{2}\right)$.

(27) If $x \in Z$, then $(r$ (the function $\exp ))^{\prime}(Z)(n)(x)=r \cdot \exp x$.

(28) $\quad\left(\square^{n}\right)_{\lceil Z}^{\prime}=\left(n\left(\square^{n-1}\right)\right)\lceil Z$.

(29) If $x \neq 0$, then $\frac{1}{\square^{n}}$ is differentiable in $x$ and $\left(\frac{1}{\square^{n}}\right)^{\prime}(x)=-\frac{n \cdot x^{n-1}}{\left(x^{n}\right)^{2}}$.

(30) If $n \geq 1$, then $\left(\square^{n}\right)^{\prime}(Z)(2)=\left((n \cdot(n-1))\left(\square^{n-2}\right)\right)\lceil Z$.

(31) If $n \geq 2$, then $\left(\square^{n}\right)^{\prime}(Z)(3)=\left((n \cdot(n-1) \cdot(n-2))\left(\square^{n-3}\right)\right)\lceil Z$.

(32) If $n>m$, then $\left(\square^{n}\right)^{\prime}(Z)(m)=\left(\left(\left(\begin{array}{c}n \\ m\end{array}\right) \cdot m !\right)\left(\square^{n-m}\right)\right) \uparrow Z$.

(33) If $f$ is differentiable $n$ times on $Z$, then $(-f)^{\prime}(Z)(n)=-f^{\prime}(Z)(n)$ and $-f$ is differentiable $n$ times on $Z$.

(34) If $x_{0} \in Z$, then (Taylor(the function $\left.\left.\sin , Z, x_{0}, x\right)\right)(n)=$ 
$\frac{\sin \left(x_{0}+\frac{n \cdot \pi}{2}\right) \cdot\left(x-x_{0}\right)^{n}}{n !}$ and (Taylor(the function $\left.\left.\cos , Z, x_{0}, x\right)\right)(n)=$
$\frac{\cos \left(x_{0}+\frac{n \cdot \pi}{2}\right) \cdot\left(x-x_{0}\right)^{n}}{n !}$.

(35) If $r>0$, then (Maclaurin(the function $\sin ,]-r, r[, x))(n)=\frac{\sin \left(\frac{n \cdot \pi}{2}\right) \cdot x^{n}}{n !}$ and (Maclaurin(the function $\cos ,]-r, r[, x))(n)=\frac{\cos \left(\frac{n \cdot \pi}{2}\right) \cdot x^{n}}{n !}$.

(36) If $n>m$ and $x \in Z$, then $\left(\square^{n}\right)^{\prime}(Z)(m)(x)=\left(\begin{array}{c}n \\ m\end{array}\right) \cdot m ! \cdot x^{n-m}$.

(37) If $x \in Z$, then $\left(\square^{m}\right)^{\prime}(Z)(m)(x)=m$ !.

(38) $\square^{n}$ is differentiable $n$ times on $Z$.

(39) If $x \in Z$ and $n>m$, then $\left(a\left(\square^{n}\right)\right)^{\prime}(Z)(m)(x)=a \cdot\left(\begin{array}{c}n \\ m\end{array}\right) \cdot m ! \cdot x^{n-m}$.

(40) If $x \in Z$, then $\left(a\left(\square^{n}\right)\right)^{\prime}(Z)(n)(x)=a \cdot n$ !.

(41) If $x_{0} \in Z$ and $n>m$, then $\left(\right.$ Taylor $\left.\left(\square^{n}, Z, x_{0}, x\right)\right)(m)=\left(\begin{array}{c}n \\ m\end{array}\right) \cdot x_{0}{ }^{n-m}$. $\left(x-x_{0}\right)^{m}$ and $\left(\right.$ Taylor $\left.\left(\square^{n}, Z, x_{0}, x\right)\right)(n)=\left(x-x_{0}\right)^{n}$.

(42) Let $n, m$ be elements of $\mathbb{N}$ and $r, x$ be real numbers. If $n>m$ and $r>0$, then $\left(\right.$ Maclaurin $\left.\left(\square^{n},\right]-r, r[, x)\right)(m)=0$ and (Maclaurin $\left.\left(\square^{n},\right]-r, r[, x)\right)(n)=x^{n}$.

(43) $\frac{1}{\square^{n}}$ is differentiable on $] 0, r[$.

(44) If $\left.x_{0} \in\right] 0, r\left[\right.$, then $\left.\left(\frac{1}{\square^{n}}\right)^{\prime}\right] 0, r\left[\left(x_{0}\right)=-\frac{n}{\left(\square^{n+1}\right)\left(x_{0}\right)}\right.$.

(45) If $x \neq 0$, then $\frac{1}{\square^{1}}$ is differentiable in $x$ and $\left(\frac{1}{\square^{1}}\right)^{\prime}(x)=-\frac{1}{\left(x^{1}\right)^{2}}$.

(46) If $] 0, r\left[\subseteq \operatorname{dom}\left(\frac{1}{\square^{2}}\right)\right.$, then $\left(\frac{1}{\square^{\mathrm{I}}}\right)_{\lceil] 0, r[}^{\prime}=\left((-1) \frac{1}{\square^{2}}\right)\lceil] 0, r[$.

(47) If $x \neq 0$, then $\frac{1}{\square^{2}}$ is differentiable in $x$ and $\left(\frac{1}{\square^{2}}\right)^{\prime}(x)=-\frac{2 \cdot x^{1}}{\left(x^{2}\right)^{2}}$.

(48) If $] 0, r\left[\subseteq \operatorname{dom}\left(\frac{1}{\square^{3}}\right)\right.$, then $\left(\frac{1}{\square^{2}}\right)^{\prime}{ }_{\uparrow 0, r[}=\left((-2) \frac{1}{\square^{3}}\right)\lceil] 0, r[$.

(49) If $n \geq 1$, then $\left.\left(\frac{1}{\square^{n}}\right)_{\uparrow] 0, r[}^{\prime}=\left((-n) \frac{1}{\square^{n+1}}\right) \uparrow\right] 0, r[$.

(50) Suppose $f_{1}$ is differentiable 2 times on $Z$ and $f_{2}$ is differentiable 2 times on $Z$. Then $\left(f_{1} f_{2}\right)^{\prime}(Z)(2)=f_{1}^{\prime}(Z)(2) f_{2}+2\left(\left(f_{1}\right)_{\uparrow Z}^{\prime}\left(f_{2}\right)_{\uparrow Z}^{\prime}\right)+f_{1} f_{2}^{\prime}(Z)(2)$.

(51) If $Z \subseteq \operatorname{dom}\left(\right.$ the function $\ln$ ) and $Z \subseteq \operatorname{dom}\left(\frac{1}{\square^{1}}\right)$, then (the function $\ln )_{\uparrow Z}^{\prime}=\frac{1}{\square^{1}}\lceil Z$.

(52) If $n \geq 1$ and $\left.x_{0} \in\right] 0, r\left[\right.$, then $\left(\frac{1}{\square^{n}}\right)^{\prime}(] 0, r[)(2)\left(x_{0}\right)=n \cdot(n+1) \cdot\left(\frac{1}{\square^{n+2}}\right)\left(x_{0}\right)$.

(53) ((The function $\operatorname{sin)~(the~function~} \sin ))^{\prime}(Z)(2)=2((($ the function cos) $($ the function $\cos ))\lceil Z)+(-2)((($ the function $\sin ) \quad($ the function $\sin ))\lceil Z)$.

(54) ((The function cos) (the function $\cos ))^{\prime}(Z)(2)=2((($ the function $\sin )$ $($ the function $\sin ))\lceil Z)+(-2)((($ the function cos $)$ (the function $\cos ))\lceil Z)$.

(55) ((The function $\operatorname{sin)~(the~function~} \cos ))^{\prime}(Z)(2)=$ $4(((-$ the function $\sin )$ (the function $\cos ))\lceil Z)$.

(56) Suppose $Z \subseteq$ dom (the function tan). Then the function tan is differentiable on $Z$ and (the function $\tan )^{\prime}{ }^{\prime}=\left(\frac{1}{\text { the function } \cos } \frac{1}{\text { the function } \cos }\right)\lceil Z$.

(57) Suppose $Z \subseteq$ dom (the function tan). Then $\frac{1}{\text { the function cos }}$ is differentiable on $Z$ and $\left(\frac{1}{\text { the function } \cos }\right)^{\prime}{ }_{\uparrow}=\left(\frac{1}{\text { the function } \cos }(\right.$ the function tan $\left.)\right) \uparrow Z$. 
(58) Suppose $Z \subseteq$ dom (the function tan). Then (the function $\tan ^{\prime}(Z)(2)=$ $2\left(\left((\right.\right.$ the function $\left.\tan ) \frac{1}{\text { the function cos the function cos }}\right)\lceil Z)$.

(59) Suppose $Z \subseteq$ dom (the function cot). Then

(i) the function cot is differentiable on $Z$, and

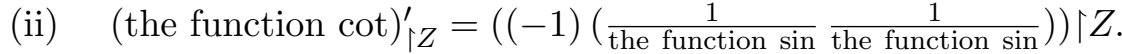

(60) Suppose $Z \subseteq$ dom (the function cot). Then

(i) $\frac{1}{\text { the function } \sin }$ is differentiable on $Z$, and

(ii) $\left(\frac{1}{\text { the function } \sin }\right)_{\uparrow Z}^{\prime}=\left(-\frac{1}{\text { the function sin }}\right.$ (the function cot $\left.)\right)\lceil Z$.

(61) Suppose $Z \subseteq$ dom (the function cot). Then (the function $\cot )^{\prime}(Z)(2)=$

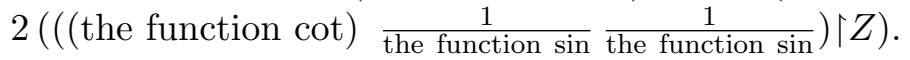

(62) $((\text { The function exp) (the function } \sin ))^{\prime}(Z)(2)=2((($ the function exp) (the function $\cos ))\lceil Z$ ).

(63) ((The function exp) (the function $\cos ))^{\prime}(Z)(2)=2(($ the function $\exp )$ -the function $\sin )\lceil Z)$.

(64) Suppose $f_{1}$ is differentiable 3 times on $Z$ and $f_{2}$ is differentiable 3 times on $Z$. Then $\left(f_{1} f_{2}\right)^{\prime}(Z)(3)=f_{1}{ }^{\prime}(Z)(3) f_{2}+\left(3\left(f_{1}{ }^{\prime}(Z)(2)\left(f_{2}\right)^{\prime}{ }^{\prime} Z\right)+\right.$ $\left.3\left(\left(f_{1}\right)^{\prime}{ }_{\lceil} f_{2}{ }^{\prime}(Z)(2)\right)\right)+f_{1} f_{2}{ }^{\prime}(Z)(3)$.

(65) ((The function sin) (the function $\sin ))^{\prime}(Z)(3)=(-8)((($ the function $\cos )$ (the function $\sin ))\lceil Z$ ).

(66) If $f$ is differentiable 2 times on $Z$, then $(f f)^{\prime}(Z)(2)=2\left(f f^{\prime}(Z)(2)\right)+$ $2\left(f_{\uparrow Z}^{\prime} f_{\uparrow Z}^{\prime}\right)$.

(67) Suppose $f$ is differentiable 2 times on $Z$ and for every $x_{0}$ such that $x_{0} \in Z$ holds $f\left(x_{0}\right) \neq 0$. Then $\left(\frac{1}{f}\right)^{\prime}(Z)(2)=\frac{2 f_{\uparrow Z}^{\prime} f_{\lceil Z}^{\prime}-f^{\prime}(Z)(2) f}{f(f f)}$.

(68) $\quad(($ The function $\exp )$ (the function $\sin ))^{\prime}(Z)(3)=(2($ (the function $\exp )$ $(-$ the function $\sin +$ the function $\cos )))\lceil Z$.

\section{REFERENCES}

[1] Grzegorz Bancerek. The fundamental properties of natural numbers. Formalized Mathematics, 1(1):41-46, 1990.

[2] Czesław Byliński. The complex numbers. Formalized Mathematics, 1(3):507-513, 1990.

[3] Czesław Byliński. Functions from a set to a set. Formalized Mathematics, 1(1):153-164, 1990.

[4] Chuanzhang Chen. Mathematical Analysis. Higher Education Press, Beijing, 1978.

[5] Krzysztof Hryniewiecki. Basic properties of real numbers. Formalized Mathematics, $1(\mathbf{1}): 35-40,1990$.

[6] Jarosław Kotowicz. Partial functions from a domain to the set of real numbers. Formalized Mathematics, 1(4):703-709, 1990.

[7] Jarosław Kotowicz. Real sequences and basic operations on them. Formalized Mathematics, 1(2):269-272, 1990.

[8] Rafał Kwiatek. Factorial and Newton coefficients. Formalized Mathematics, 1(5):887-890, 1990.

[9] Akira Nishino and Yasunari Shidama. The Maclaurin expansions. Formalized Mathematics, 13(3):421-425, 2005.

[10] Beata Perkowska. Functional sequence from a domain to a domain. Formalized Mathematics, 3(1):17-21, 1992. 
[11] Konrad Raczkowski. Integer and rational exponents. Formalized Mathematics, 2(1):125130, 1991.

[12] Konrad Raczkowski and Paweł Sadowski. Real function differentiability. Formalized Mathematics, 1(4):797-801, 1990.

[13] Konrad Raczkowski and Paweł Sadowski. Topological properties of subsets in real numbers. Formalized Mathematics, 1(4):777-780, 1990.

[14] Yasunari Shidama. The Taylor expansions. Formalized Mathematics, 12(2):195-200, 2004.

[15] Andrzej Trybulec and Czesław Byliński. Some properties of real numbers. Formalized Mathematics, 1(3):445-449, 1990.

[16] Zinaida Trybulec. Properties of subsets. Formalized Mathematics, 1(1):67-71, 1990.

[17] Edmund Woronowicz. Relations defined on sets. Formalized Mathematics, 1(1):181-186, 1990.

[18] Yuguang Yang and Yasunari Shidama. Trigonometric functions and existence of circle ratio. Formalized Mathematics, 7(2):255-263, 1998.

Received March 18, 2008 